\title{
Optimization of Community Based Virtual Power Plant with Embedded Storage and Renewable Generation
}

\author{
Oghenovo Okpako*, Paul Inuwa Adamu, Haile-Selassie Rajamani, and Prashant Pillai \\ Faculty of Engineering and Informatics, \\ University of Bradford, UK \\ \{ookpako, p.i.adamu, H.S.Rajamani, P.Pillai\} @bradford.ac.uk
}

\begin{abstract}
The current global challenge of climate change has made renewable energy usage very important. There is an ongoing drive for the deployment of renewable energy resource at the domestic level through feed-in tariff, etc. However, the intermittent nature of renewable energy has made storage a key priority. In this work, a community having a solar farm with energy storage embedded in the house of the energy consumers is considered. Consumers within the community are aggregated in to a local virtual power plant. Genetic algorithm was used to develop an optimized energy transaction for the virtual power plant with respect to differential pricing and renewable generation. The results show that it is feasible to have a virtual power plant setup in a local community that involve the use of renewable generation and embedded storage. The results show that both pricing and renewable generation window should be considered as a factor when setting up a virtual power plant that involve the use of storage and renewable generation at the community level. Also, when maximization of battery state of charge is considered as part of an optimization problem in a day ahead market, certain trade-off would have to be made on the profit of the virtual power plant, the incentive of the prosumer, as well as the provision of peak service to the grid.
\end{abstract}

Keywords: Prosumer; Battery; Virtual Power Plant (VPP); Genetic Algorithm (GA); Smart Grid, State of charge; Solar Generation.

\section{Introduction}

Access to clean energy is becoming important. This is due to the current global challenge of climate change caused by the emission of greenhouse gases from fossil fuel. The need for clean energy has ensured that vital consideration is given to energy produced from renewable energy sources. There is a current drive to promote much use of renewable energy, for example, the European Union has renewable energy usage as part of its strategy to cut down greenhouse gas emission by $80 \%$ by the year 2050 [1]. Also, most governments are currently encouraging the use of renewable energy through the provision of feed-in tariff, etc. However, renewable energy sources like solar, wind etc. are intermittent in nature, and would require energy storage devices. Energy storage becomes key in maximizing the use of renewable energy. It can be used to smoothen peak and trough of renewable generation.

There is a current global restructuring of electric power utilities [2], [3]. This is envisaged to change the consumer role to that of a prosumer, and as well promote the deployment of distributed generation. The prosumer role involves both energy consumption and energy production. Energy storage becomes an asset to the prosumer as it could be to buy cheap energy from the grid during off-peak period, or store excess renewable generation, which could later be sold at peak period for better prices [4], [5], [6].

The concept of using small unit of energy storage at the domestic side of the electricity grid to participate in the power market was proposed by Kempton [7]. According to Kempton, the battery electric vehicle is not just a load on the utility grid, but an alternative power source. Work on different energy management strategies for dealing with battery electric vehicles has been done by these authors [8], [9], [10]. However, battery electric vehicles are usually mobile and could be limited in their potentials to maximize the use of renewable energy. As a result, it is proposed in this work to have battery energy storage embedded inside the home of the prosumer.

At the domestic side of the grid, prosumers lack the capacity to participate directly at the wholesale power market. Prosumers would need to be aggregated by a virtual power plant (VPP) at the community level. The VPP aggregates small units of distributed energy resource and flexible load for participation in the power market by offering flexibility. Prosumers participation in the power market is done through the VPP. In [11], it is demonstrated that it is possible to have a pricing scheme that provides financial incentive for both the VPP and the prosumer. However, there was no clarity on how VPP operation is affected with both pricing and renewable energy generation. Also the possibility and effect of including battery state of charge as part of an optimization problem for a community VPP was clearly not understood. 
In this paper, it is proposed to use genetic algorithm to optimize the energy transaction of a community VPP with respect to differential pricing and renewable generation. The algorithm was tested under various scenarios. These includes an objective function where only the prosumer net cost is considered, and also an objective function where both the prosumer net cost and battery state of charge were considered.

This paper is organized as follows; section 2 is a description of the model, section 3 is the mathematical modelling, section 4 is the VPP optimization, section 5 is the results and discussion, and section 6 is the conclusion.

\section{Framework of Virtual Power Plant Model}

Fig. 1, is a diagram describing the VPP model developed in this work.

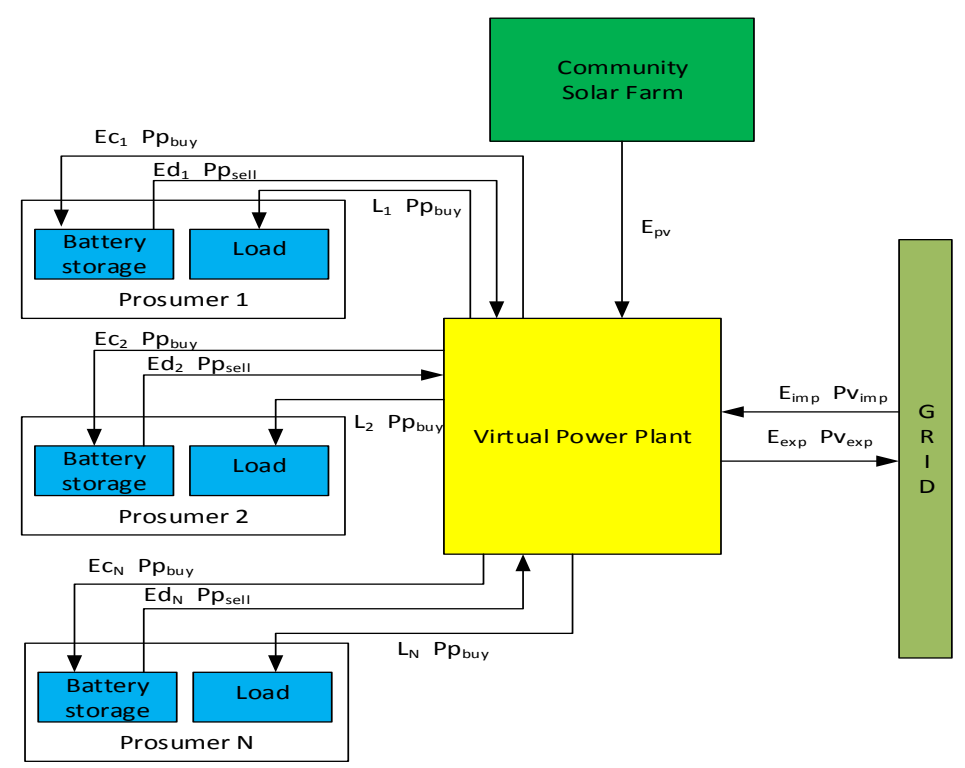

Fig. 1. Architecture of the Virtual Power Plant Model.

From Fig. 1, $\mathrm{N}$ is the total number of prosumers within the community aggregated as a VPP. $\operatorname{Ed}_{1}$ to $\mathrm{Ed}_{\mathrm{N}}$ is the discharge energy from prosumer 1 to $\mathrm{N}$ battery. $E c_{1}$ to $E c_{N}$ is the charge energy for prosumer 1 to $\mathrm{N}$ battery. $\mathrm{Pp}_{\text {sell }}$ is the prosumer sell price of energy from battery, or the price at which the VPP buys energy from the prosumer's battery. $\mathrm{L}_{1}$ to $\mathrm{L}_{\mathrm{N}}$ is the load demand of prosumer 1 to $\mathrm{N}$. $\mathrm{Pp}_{\text {buy }}$ is the price at which the prosumer buy energy from the VPP to meet its load, or the price at which VPP sells energy to the prosumer to meet load demand. $E_{i m p}$ and $E_{\text {exp }}$ are the amount of energy imported from the grid, and exported to the grid by the VPP. $P v_{\text {imp }}$ and $P v_{\text {exp }}$ are the VPP import and export price of energy to the grid. Epv is the solar energy generation from the community solar farm which is assumed to be free for both parties (prosumer and VPP).

\subsection{Virtual Power Plant}

In Fig. 1, the VPP can buy energy in bulk from the grid $\left(E_{\text {imp }}\right)$ at price $P v_{\text {imp }}$ and from the prosumers $\left(E_{1}\right.$ to $E d_{N}$ from prosumer 1 to prosumer N) at price Prsell respectively. The energy bought from the grid is use to meet the prosumer's energy demand $\left(\mathrm{L}_{1}\right.$ to $\left.\mathrm{L}_{\mathrm{N}}\right)$ as well as to charge their battery. The energy bought from each prosumer's battery $\left(\mathrm{Ed}_{1}\right.$ to $\mathrm{Ed}_{\mathrm{N}}$ from prosumer 1 to prosumer $\left.\mathrm{N}\right)$ are aggregated by the VPP. The aggregated energy is first used within the community to meet each prosumer's load demand respectively before its excess can be sold to the VPP and then exported (traded in the power market). The solar farm is owned by the community. Energy produced from the solar farm is only use for charging of the prosumers battery and meeting of their load demand. The VPP only imports energy from the external grid when the energy produced from the community solar farm is not enough to meet the prosumers battery charging and load requirement.

In this work, the VPP was considered as having a day ahead forecast of the energy produced from the community solar farm. The VPP has a day ahead forecast of each prosumer half hourly load profile. In addition, the VPP has a day ahead forecast of the price Pvexp at which the external grid would buy its energy (i.e. the day 
ahead forecast price paid by the grid to the VPP for exporting energy), as well as the day ahead forecast of the price Pvimp at which the grid would sell energy to the prosumer (i.e. the day ahead forecast price paid by VPP to the external grid for importing energy). Both import and export prices for energy are agreed between the VPP and the grid in the wholesale power market. Based on the day ahead import and export price, the VPP agrees a day ahead prosumer buy and sell price of energy. Thereafter, the VPP has to optimally allocate energy resource by determining its day ahead schedule assuming no error band during forecasting. The day ahead energy resource allocation is done by determining the day ahead charge/discharge energy from each prosumer battery. The charge/discharge energy is use to control the amount of energy to be imported from grid and exported to the grid by the community, which is subject to the availability of energy from the community solar farm. The incentives for encouraging the VPP as a business entity is profit.

\subsection{Prosumer}

A community consisting of three prosumers $(\mathrm{N}=3)$ was considered in this model. Each prosumer was considered as having battery energy storage embedded inside their home. Each battery is considered as having a state of charge of $30 \%$ respectively. The day ahead half hourly load profile of each prosumer is shown in Fig. 2. Fig. 2, is a typical half- hourly load profile of three different class of domestic energy consumers within a residential community. This data was obtained from Xcel Energy [12].

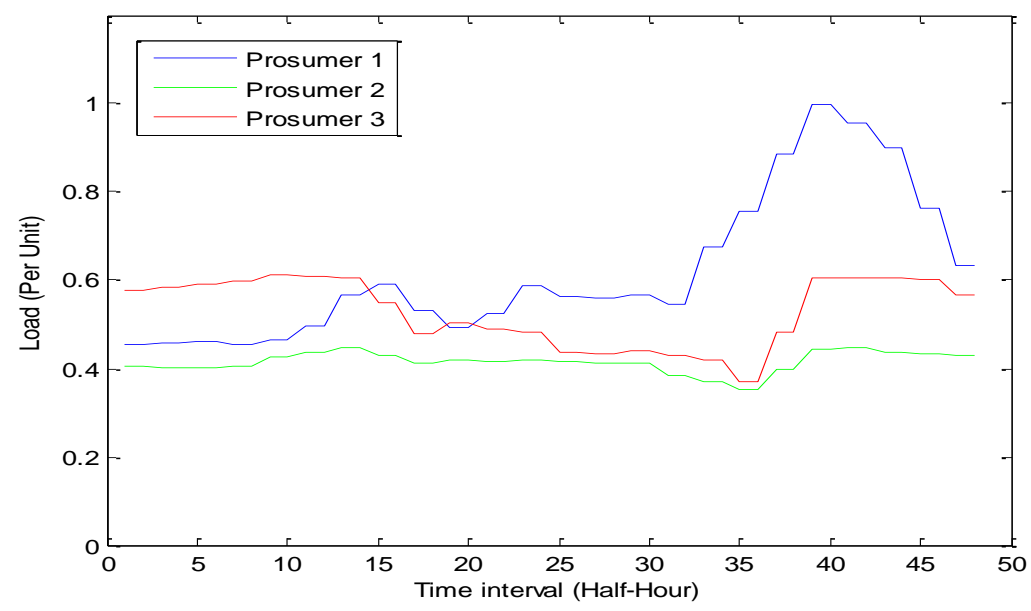

Fig. 2. Forecasted hourly load profile of each prosumer.

Each of the prosumers has a different hourly load profile. The motivation of the prosumer as a participant in the VPP, is to minimize its net cost (energy purchasing cost). In this work, a lower net cost represents an incentive received by the prosumer for participating as part of a VPP in its local community.

\section{Mathematical Tool used in Modelling}

\subsection{The VPP Energy Balance}

The VPP energy balance equation during the time interval $t$ is calculated in (1) as follows.

$$
\begin{gathered}
E_{i m p_{t}}+E_{p v_{t}}+\sum E_{d_{i, t}}=E_{\exp _{t}}+E_{n p v_{t}}+\sum\left(E_{c_{i, t}}+L_{i, t}\right) \\
\left\{\begin{array}{l}
\text { if }\left(E_{p v_{t}}+\sum\left(E_{d_{i, t}}-E_{c_{i, t}}-L_{i, t}\right)<0, E_{i m p_{t}}=+v e\right. \\
\text { if }\left(E_{p v_{t}}+\sum\left(E_{d_{i, t}}-E_{c_{i, t}}-L_{i, t}\right)>0\right), E_{i m p_{t}}=0, E_{n p v_{t}}=+v e \\
\text { if }\left(\sum\left(E_{d_{i, t}}-L_{i, t}\right)\right)>0, E_{\exp _{i, t}}=+v e \\
\text { if }\left(\sum\left(E_{d_{i, t}}-L_{i, t}\right)\right)<0, E_{\exp _{i, t}}=0
\end{array}\right.
\end{gathered}
$$


Where $i$ is an integer. $t$ is the time interval. $E_{i m p_{t}}$ and $E_{\text {exp }}$ are the amount of import energy and the amount of export energy in per unit during t. $E_{c_{i, t}}$ and $E_{d_{i, j}}$ are the amount of charge and discharge energy in per unit respectively allocated to prosumer $i$ battery by the VPP during $t$. $L_{i, t}$ is the load of prosumer $i$ in per unit during $t$. The load is fixed. $E_{p v_{t}}$ is the amount of energy in per unit produced from community solar farm. $E_{n p u_{t}}$ is the amount of energy produced from the community solar farm that is not used by the community.

\section{2}

VPP Profit

The VPP profit $V p p_{\text {profit }}$, at each time interval $t$ over the day's total number of time interval $(T)$ is calculated as follows.

$$
\sum_{t=1}^{T} V p p_{\text {profit }_{t}}=\sum_{t=1}^{T}\left(V p p_{\text {rev }_{t}}-V p p_{\cos t_{t}}\right)
$$

Where $V p p_{\text {rev }_{t}}$ and $V p p_{\text {cost }_{t}}$ are the VPP revenue and cost respectively during the time interval $t$. $T$ is the day's total number of time interval. Both VPP revenue and cost are calculated based on the amount of energy imported and exported. This is because it does not cost the VPP to get energy from the solar farm, also the prosumers only sells excess energy to the VPP for export after their load demand have been met. VPP revenue and cost are calculated in (3) and (4) respectively as follows.

$$
\begin{gathered}
\sum_{t=1}^{T} V p p_{r e v_{t}}=\sum_{t=1}^{T} P p_{b u y_{y^{\prime}}} \cdot E_{i m p_{t}}+P v_{\text {exp }} \cdot E_{\text {exp }_{t}} \\
\sum_{t=1}^{T} V p p_{\cos t_{t}}=\sum_{t=1}^{T} P v_{i m p_{t}} \cdot E_{i m p_{t}}+P p_{\text {sell }_{t}} \cdot E_{\exp _{t}}
\end{gathered}
$$

Where $P p_{\text {sell }}, P p_{b y_{1}}, P v_{\text {imp }}$, and $P v_{\text {exp }}$ are the prosumer selling price of energy, prosumer buy price of energy, the VPP import price of energy, and the VPP export price of energy respectively during $t$. All measured in pence/per unit.

\subsection{Prosumer Net Cost}

The prosumer net cost is calculated using both the import and export energy. During energy import, only energy imported from the grid is paid for by the prosumer. Energy used from the community solar farm is not paid for by the prosumer. The import energy represents a deficit in energy production from the community solar farm. During energy export, the energy discharge from the prosumer battery is first used internally to meet the prosumer's load demand, only its excess is sold to the VPP for export. The prosumer's net cost $P p_{\text {cost }}$, at each time interval $t$ over $T$ is calculated as follows.

$$
\sum_{t=1}^{T} P p_{\cos _{t}}=\sum_{t=1}^{T} P p_{b u y_{t}} \cdot E_{i m p_{t}}-P p_{s e l l_{t}} \cdot E_{\exp _{t}}
$$

\subsection{Battery State of Charge.}

The battery state of charge (SOC) gives an information on the battery energy level. In this work, the battery energy level is measured in per unit. Usually the battery SOC cannot be measured directly, but can be inferred from the battery energy level. Therefore, the battery state of charge of charge is a measure of the battery energy level in comparison to the battery actual capacity, assuming an ideal battery with no peukert effect, no losses (self-discharge) and whose actual capacity is the same as its nominal capacity. The SOC is measured in percentage. It gives an information on the battery depth of discharge. The battery energy level measured during $t$ is calculated as follows.

$$
E_{\text {stored }_{i, t}}=E_{o_{i}}+\sum_{t=1}^{T} E_{c d}
$$




$$
E c d_{i, t}=\left\{\begin{array}{l}
E_{c_{i, t}}, \text { if battery charg ing occur } \\
-E_{d_{i,}}, \text { if battery disch } \arg \text { ing occur } \\
0, \text { if battery is idle }
\end{array}\right.
$$

$E_{\text {stored }_{i, t}}$ is prosumer $i$ battery energy level in per unit measured at $t . E_{o_{i}}$ is prosumer $i$ initial battery energy level in per unit before participation in the day ahead power market. Each prosumer battery SOC at $t$ is calculated as follows.

$$
S O C_{i, t}=100 \frac{E_{\text {stored }_{i, t}}}{E_{\text {batt }_{i}}}
$$

$S O C_{i, t}$ is the state of charge of prosumer $i$ battery measured in percentage at $t . E_{b a t t_{i}}$, is the actual battery capacity in per unit of prosumer $i$.

\subsection{Battery Constraints}

Each prosumer battery discharge constraint is represented as follows.

$$
E_{d, \min _{i}} \leq E_{d_{i, t}} \leq E_{d, \max _{i}}
$$

Where $E_{d, \min _{i}}$ and $E_{d, \max _{i}}$ are the minimum and maximum discharge energy that can be allocated to prosumer $i$ battery. Each prosumer battery charge constraint is represented as follows.

$$
E_{c, \min _{i}} \leq E_{c_{i, t}} \leq E_{c, \max _{i}}
$$

Where $E_{c, \min _{i}}$ and $E_{c, \max _{i}}$ are the minimum and maximum charge energy that can be allocated to prosumer $i$ battery. Each prosumer battery state of charge constraint is represented as follows.

$$
S O C_{\min _{i}} \leq S O C_{i, t} \leq S O C_{\max _{i}}
$$

Where $S O C_{\min _{i}}$ and $S O C_{\max _{i}}$ are the minimum and maximum SOC limit of prosumer $i$ battery.

\section{Optimizations of the Community Virtual Power Plant}

To understand the optimization problem, the number of prosumer chosen to participate in the community VPP was kept at three. The optimization problem is formulated considering both the prosumer net cost and the battery SOC. This is gotten from (5) and (7) and is represented as follows.

$$
[\text { Min }] F=w 1 \sum_{t=1}^{T} P p_{\cos t_{t}}-w 2 \sum_{t=1}^{T} \sum_{i=1}^{N} S O C_{i, t}
$$

$F$ is the objective function to be minimize. It represents both the prosumer net cost and the battery state of charge. $w 1$ and $w 2$ are the weighting factor. $E_{o}$ was chosen to be 5.4 per unit. $T$ was chosen to be 48 . Ebatt (actual battery capacity) was chosen to be 18 per unit. Given the day ahead pricing, solar generation, and prosumer's load profile, genetic algorithm was used to solve the optimization problem [11].

\section{Results and Discussion}

In Fig. 3, is the modified pricing scheme used by the VPP in this work [11], [13]. The modified pricing scheme was extended to 48 time intervals. It was considered to use the modified pricing scheme because its business incentives favors both the VPP and the prosumer [11], [13] while meeting the VPP objective. This is detailed in [11], [13]. 


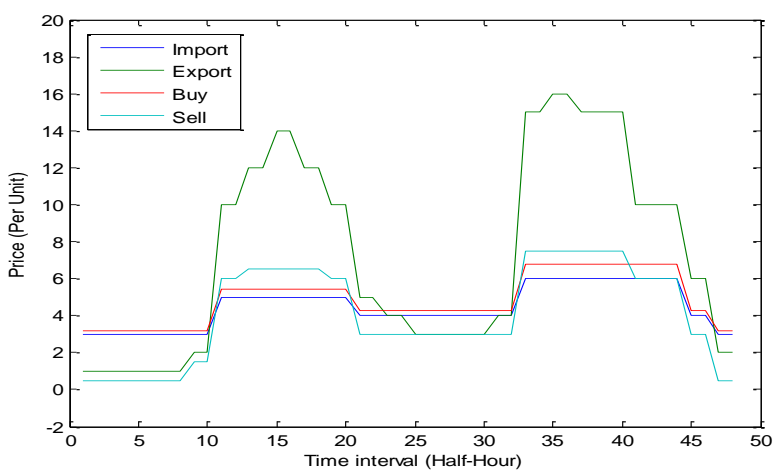

Fig. 3. Modified pricing scheme.

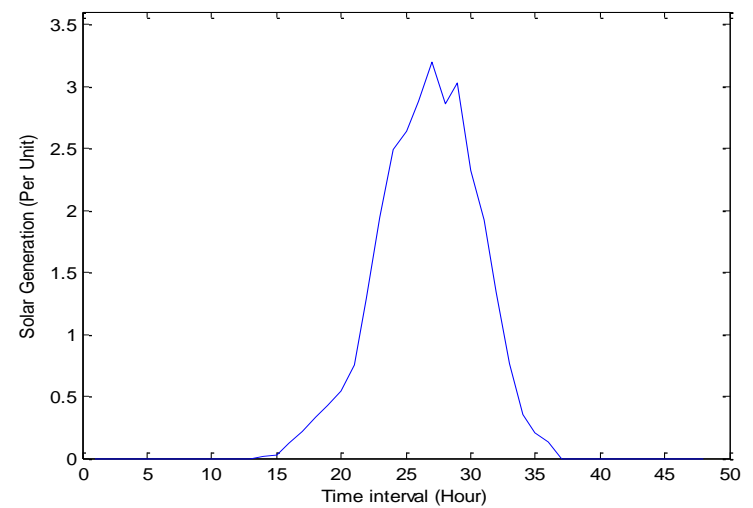

Fig. 4. Day ahead solar generation from community solar farm.

Fig. 4, is the day ahead solar generation from the community solar farm. The data was gotten from [14], and was normalized to a peak solar generation of 3.2 per unit. This would be a more realistic value for the community considered (considering their load and charging requirements).

The modified pricing scheme, solar generation, and load profile were used as the input data. GA was used to perform the optimization and was initially tested by selecting $w 1=1$ and $w 2=0$. The simulation was done with these weighting factor, and the results are presented as follows.

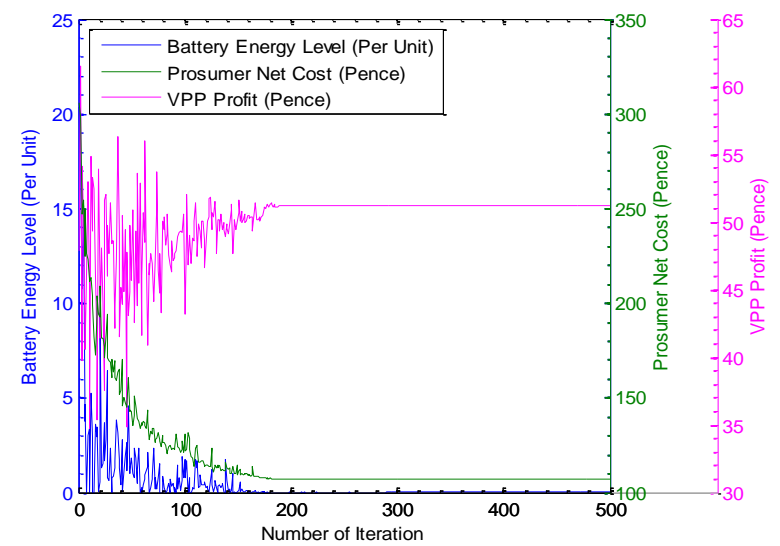

Fig. 5. Effect of optimization on community (at $w 1=1, w 2=0$ ).

In Fig. 5, the prosumer net cost decreases as the algorithm optimizes until it converges. Also, the VPP makes profit. Though the objective function with the given weighting factor purely considers the prosumers net cost minimization, the modified pricing scheme favors both the prosumer and the VPP. 


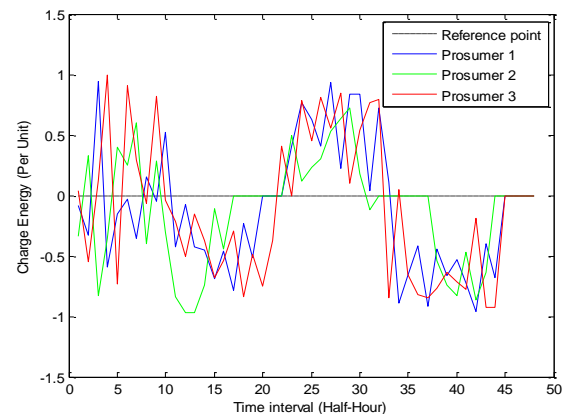

Fig. 6. Battery charge.

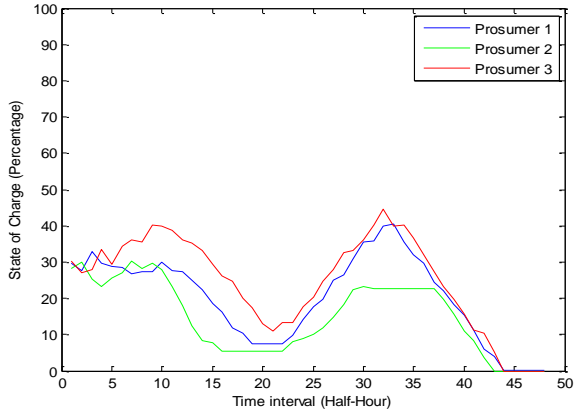

Fig. 7. Battery state of charge.

In Fig. 6, the area above the reference point represents the charge energy while the one below the reference point represent the discharge energy. It is noticed that the batteries are charging during the period of solar generation and are discharging during the grid's peak demand. This is good, because the prosumers are actually using the energy produced from solar generation for charging of their battery, and are also discharging their battery during peak period to support their load. In Fig. 7, it is observed that the battery state of charge at the end of the day is zero percent. This is not good for the battery life and state of health. This is attributed to the optimization problem which only considers the prosumer net cost for one day period. Fig. 8, is the energy exchange between the community and the grid.

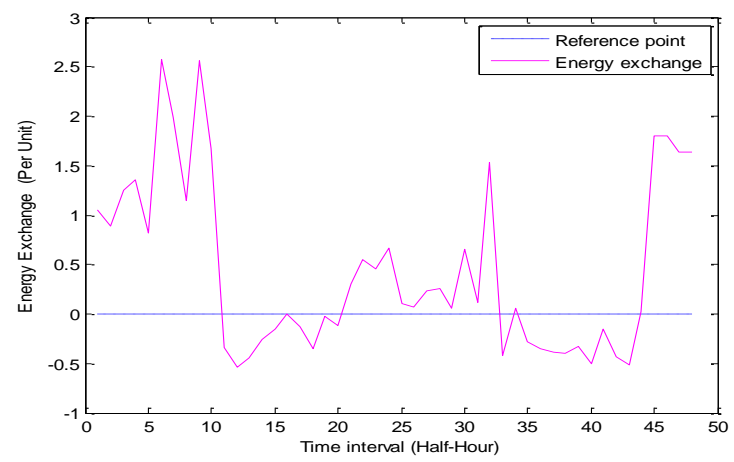

Fig. 8. Energy exchange between community and external grid.

In Fig. 8, the area above the reference point is the import energy, while below the reference point is the export energy. It is noticeable that the community is actually importing energy from the grid during off-peak period. The energy imported from the grid during the off-peak period is much lower when energy from community solar farm is available. This import is because the total load and the charging requirement of the community is slightly higher than the energy produced from the community solar farm. During the peak period, energy is exported to the external grid for grid peak support. Both peak and off-peak scenarios are good for the grid in terms of energy balancing.

However, as earlier discussed, the battery SOC is still an issue. To address this low battery SOC issue, the optimization was then run with $w 1=0.2$ and $w 2=0.8$. The results obtained are presented as follows.

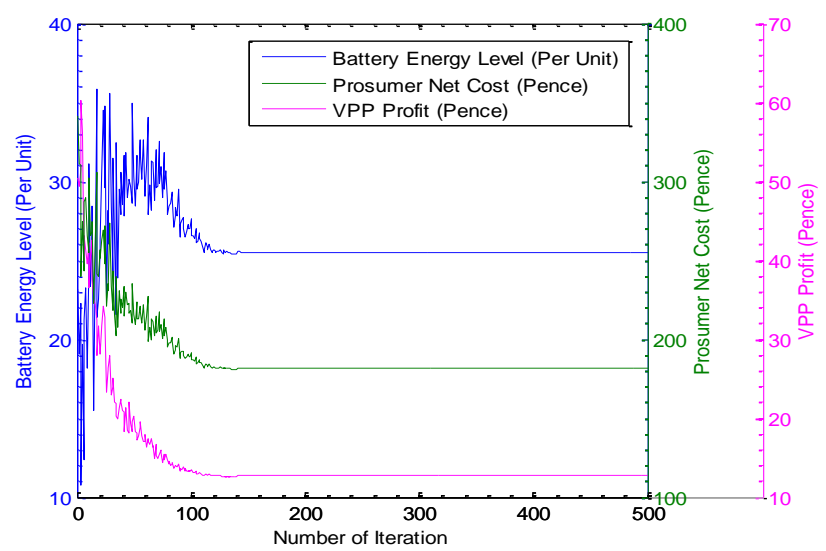


Fig. 9. Effect of optimization on community (at $w 1=0.2, w 2=0.8$ ).

In Fig. 9, when compared to Fig. 5, it is noticed that the battery energy level has increased. The prosumer net cost is higher and the VPP profit is lower. This is because of the battery SOC which was considered as part of the optimization function. Fig. 10, and Fig. 11. are the battery charge and state of charge respectively.

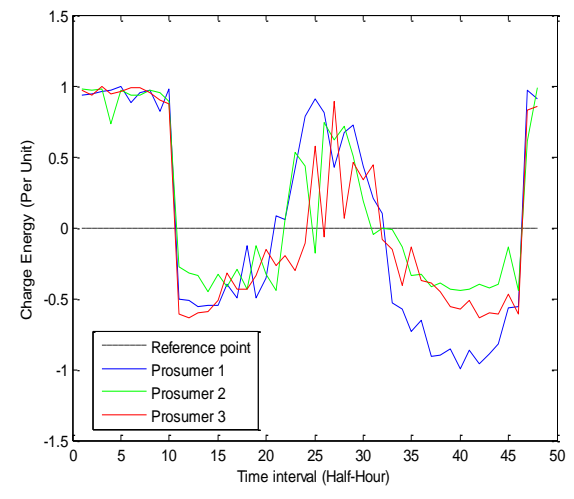

Fig. 10. Battery charge.

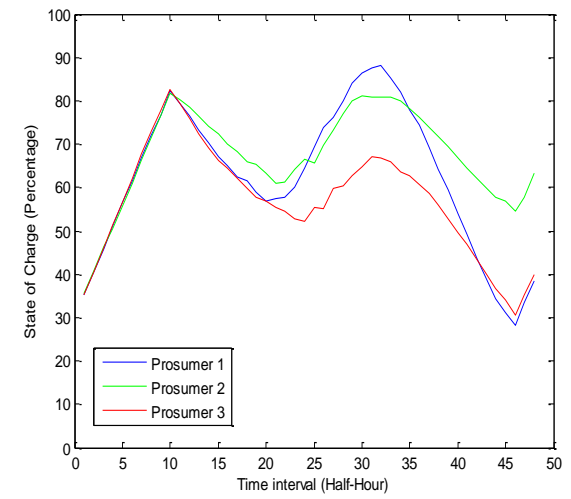

Fig. 11. Battery state of charge.

In Fig. 10, the batteries are actually charging during the grid off-peak period and during the period of renewable energy generation. This is good for the grid as grid off-peak service is provided. Also, it is good for the community as renewable energy produced from the community solar farm is utilized for charging the batteries. In Fig. 11, it is observed that the battery SOC are much higher compared to that observed in Fig. 7. This is because maximization of the battery SOC was formulated as a part of the objective function. One noticeable observation is that the SOC of prosumer 2 is much higher than others. This is because of its lower load demand. The energy exchange between the community and the grid is shown in Fig. 12.

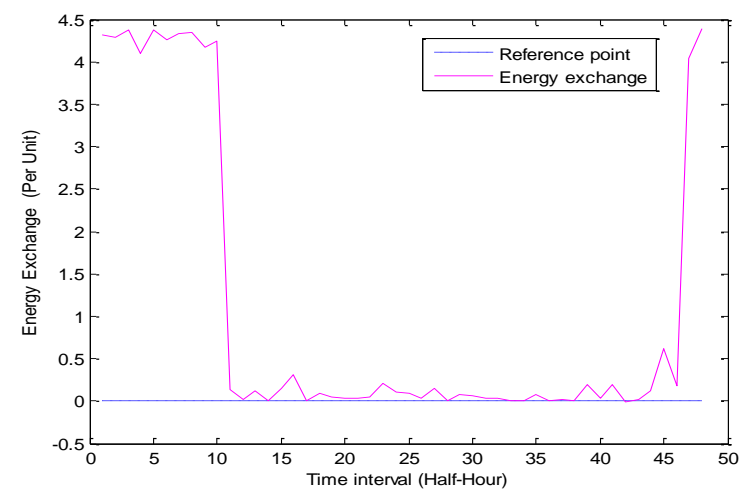

Fig. 12. Energy exchange between community and external grid.

In Fig. 12, it is observed that energy is imported from the grid during the off-peak period. Also, during the period of renewable generation, very little or no amount of energy is imported from the grid. However, during the peak period, energy is not exported to the grid, though the prosumer batteries are discharging. The discharge energy is used within the community to meet the prosumer load demand. This is attributed to the battery SOC maximization that was included as part of the objective function as well as the battery initial SOC.

This experiment has shown the feasibility of setting up a virtual power plant in a local community that would involve renewable generation and embedded storage. It has been demonstrated that both pricing and solar generation window can affect the technical operation of the VPP in terms of the charge and discharge time period as well as the amount of energy imported from the external grid. Also, it has been shown with clarity that preventing the battery from attaining a very low SOC is possible for a one-day optimization period. However, certain tradeoff would have to be made on the prosumer's incentive, VPP profit, and grid peak/offpeak support. 


\section{Conclusion}

In this paper, it has been demonstrated that it is possible to have a virtual power plant set up that involves the use of embedded energy storage and renewable generation at a community level. It has been demonstrated that both pricing and solar generation window can affect the technical operation of the VPP in terms of the charge and discharge time period as well as the amount of energy imported from the external grid. It has been shown that battery state of charge should be accounted for in an optimization algorithm particularly for day ahead market. However, in considering battery state of charge as part of an optimization problem, certain tradeoffs in terms of provision of grid peak/off-peak support and incentives for the prosumer and the virtual power plant would have to be made.

\section{Reference}

1. Carvalho, M.G.: EU energy and climate change strategy. Journal of Energy, vol. 40, no. 1, pp. 19-22. (2012)

2. Vallvé, X., Graillot, A., Gual, S., Colin, H.: Micro storage and demand side management in distributed PV gridconnected installations. In: 9th IEEE International Conference on Electrical Power Quality and Utilisation 2007, pp. 1-6. (2007)

3. Ihbal, A., Rajamani, H., Abd-Alhameed, R., Jalboub, M., Elmeshregi, A., Aljaddal, M.: Development of Electricity Pricing Criteria at Residential Community Level. Universal Journal of Electrical and Electronic Engineering, vol. 2, no. 2, pp. 81-89. (2014)

4. Ferreira, H.L., Garde, R., Fulli, G., Kling, W., Lopes, J.P.: Characterisation of electrical energy storage technologies. Journal of Energy, vol. 53, pp. 288-298. (2013)

5. Boicea, V. A.: Energy Storage Technologies: The Past and the Present. Proceedings of the IEEE, vol. 102, no. 11, pp. 1777-1794. (2014)

6. IEA.: Technology roadmap: energy storage. Paris (2014)

7. Kempton, W., Letendre, S. E.: Electric vehicles as a new power source for electric utilities. Transportation Research Part D: Transport and Environment, vol. 2, no. 3, pp. 157-175. (1997)

8. Wu, D., Aliprantis, D.C., Ying, L.: Load scheduling and dispatch for aggregators of plug-in electric vehicles. IEEE Transactions on Smart Grid, vol. 3, no. 1, pp. 368-376. (2012)

9. Pang, C., Aravinthan, V., Wang, X.: Electric vehicles as configurable distributed energy storage in the smart grid. In: Power Systems Conference (PSC) 2014, Clemson University, pp. 1-5. (2014)

10. Brooks, A., Lu, E., Reicher, D., Spirakis, C., Weihl, B.: Demand dispatch. IEEE Power and Energy Magazine, vol. 8, no. 3, pp. 20-29. (2010)

11. Okpako, O., Rajamani, H., Pillai, P., Anuebunwa, U., Swarup, K.: Evaluation of community virtual power plant under various pricing schemes. In: Proceedings of 2016 IEEE Smart Energy Grid Engineering Conference, Oshawa Canada, pp. 72-78. (2016)

12. Xcel Energy Hourly Load Profile, https://www.xcelenergy.com/staticfiles/xe/Corporate/Corporate\%20PDFs/AppendixD-Hourly_Load_Profiles.pdf

13. Okpako, O., Rajamani, H., Pillai, P., Anuebunwa, U., Swarup, K.: Investigation of an Optimized Energy Resource Allocation Algorithm for a Community Based Virtual Power Plant. In: Proceedings of 2016 IEEE PES Power Africa Conference, Livingstone - Zambia, pp. 153-157. (2016)

14. The University of Sheffield Solar, http://www.solar.sheffield.ac.uk/pvlive 